\title{
La supuesta neutralidad de la evaluación por competencias
}

\author{
Francisco José Tovar Martinez; Juan Carlos Revilla Castro \\ Universidad Complutense de Madrid \\ ftovar@cps.ucm.es; jcrevilla@cps.ucm.es
}

Resumen: La gestión por competencias se ba convertido en los ültimos treinta años en uno de los temas recurrentes cuando se babla de la gestión integral en las organizaciones del siglo XXI. Los cuatro pilares sobre los que descansa dicha gestión son la selección, la formación, la retribución y la evaluación. El objetivo del presente artículo es el análisis de uno de esos elementos clave: la evaluación por competencias. El punto de partida de esta investigación es la tendencia, en aumento, a valorar a los trabajadores tanto por sus cualificaciones como por sus actitudes, dos elementos centrales en la definición de competencia. A través del análisis de diferentes entrevistas a expertos y encargados de recursos bumanos implicados en la gestión por competencias, se pretende visualizar las dificultades de encontrar elementos objetivos a los que atenerse a la hora de evaluar las competencias de los trabajadores.

Palabras clave: gestión por competencias, subjetividad, evaluación, trabajadores e incertidumbre.

\begin{abstract}
In the last 30 years competence-based management has become a recurring theme in the integral management of 21 st-century institutions. The four pillars that support competence-based management are selection, training, remuneration and assessment. The aim of the present article is to analyse one of these key elements: competence-based assessment. The starting point of the study is the increasing tendency to assess employees on the basis not only of their qualifications but also of their attitudes, two elements that are fundamental to the definition of competence. By analyzing a variety of interviews with experts and people in charge of buman resources involved in competence-based management, we aim to highlight how difficult it is to find objective elements on which to base an assessment of the competences of employees.
\end{abstract}

Key words: competence-based management, subjectivity, assessment, workers and uncertainty. 


\section{Introducción}

La manera de gestionar las empresas se ha diversificado en los últimos veinticinco años. Dicha diversificación ha ido de la mano del aumento de organizaciones vinculadas a la sociedad del conocimiento, es decir, organizaciones donde la mano de obra ha ido adquiriendo un papel más secundario para dar paso a la cabeza en el proyecto, o lo que es lo mismo, poner mayor énfasis en el valor de las personas. Dichas organizaciones se caracterizan por las prácticas de trabajo en grupos, la autonomía de los puestos de trabajo, la reducción de niveles jerárquicos, la integración de tareas y la subcontratación y descentralización de actividades (Martín Artiles, 2005).

En ese nuevo contexto, las organizaciones esprintan por conseguir la ventaja competitiva ahí donde saben que nadie les puede copiar, su capital humano. Conseguir a los individuos mejor capacitados y formarles para que evolucionen a la misma velocidad que cambia el mercado son los objetivos principales. Por ello, los departamentos denominados de recursos bumanos han pasado de ser departamentos secundarios a convertirse en esenciales (Pralahad y Hammel, 1990; Spencer y Spencer, 1993), pues se considera que en la gestión de las personas reside el devenir de la empresa. La gestión por competencias se sitúa como la piedra angular a partir de la cual articular las funciones de reclutamiento, selección, promoción, remuneración y evaluación de los miembros de la organización, de forma que se ha convertido en una máquina eficaz al servicio de la competitividad económica a través de la adaptación y la flexibilidad (Brunet, 2005).

La gestión por competencias sería en las organizaciones la expresión última de lo que diversos autores denominan la gestión flexible del empleo (Brunet, 2005; Serrano y Crespo, 2002), al implicar la desaparición del convenio colectivo como contrato regulador entre empresario y trabajadores, y, prácticamente, del reconocimiento curricular. El trabajador, en este contexto, ha visto cómo la organización deja de otorgar importancia a los certificados que posee para pasar a considerar imprescindible una buena cartera de competencias. Éstas se tienen o no en la práctica, es decir, en la demostración empírica de que sabe desarrollar su trabajo. Las competencias no abarcan sólo la esfera técnica del desarrollo de la tarea, sino que, además, implican los aspectos de corte más psicológico de la persona. Las competencias personales son consideradas por la organización como necesarias para el desarrollo correcto de la tarea. En ese sentido, la organización demanda al individuo no solamente sus conocimientos técnicos en un determinado campo, sino también sus comportamientos, actitudes y motivaciones.

La evaluación por competencias en las organizaciones implica la evaluación de competencias técnicas que están estrechamente vinculadas a la formación del individuo y de competencias generales o conductuales. ${ }^{1}$ En ese sentido, existiría una dicoto-

1 La utilización de las expresiones competencia general o competencia conductual depende totalmente de los autores y las empresas a los que se recurra. Cada cual utiliza un nombre para hacer referencia a los comportamientos, los rasgos, las 
mía evaluadora, ya que para las primeras, las técnicas, es posible encontrar evidencias que permitan evaluar de forma más o menos objetiva el desempeño del individuo; en cambio, dicha evaluación objetiva es mucho menos evidente en las generales, a pesar de que en ningún manual de gestión de competencias aparezca tal cuestión. La evaluación adquiere un protagonismo fundamental en el devenir laboral del individuo al posicionarlo en un determinado nivel de cualificación a partir del cual le será posible conocer las diferentes alternativas laborales dentro de la organización en los años siguientes.

El presente artículo comenzará esbozando una idea general de qué significa e implica la gestión por competencias en las organizaciones actuales, poniendo especial énfasis en la evaluación por competencias y describiendo sus principales herramientas y características. En un segundo punto se analizará el discurso de cinco empresas españolas de diferentes sectores con gestión por competencias a través de sus dirigentes de recursos humanos y de documentos corporativos. Su discurso se desglosará en función de tres puntos. El primero tratará sobre los esfuerzos de los diferentes actores para definir la noción de competencia. El segundo tratará de mostrar cómo dichos actores buscan una objetividad en la evaluación de sus empleados que muchas veces parece imposible, y el tercer eje discursivo, en tensión con el anterior, explicitará la importancia del aspecto subjetivo a la hora de evaluar. El resultado de lo anterior indica la falta de criterios claros a la hora de evaluar el desempeño de los trabajadores, cuya valoración deja de ser el reflejo de su esfuerzo laboral a lo largo del año y en la que, cada vez más, aumentan variables de corte más psicológico (Crespo, Revilla y Serrano, 2007; Tovar y Revilla, 2009).

\section{La gestión por competencias}

Los principales manuales manageriales (Spencer y Spencer, 1994; Dalziel, Cubeiro y Fernández, 1996; Fernández López, 2006) presentan la gestión por competencias como la mejor respuesta al cambio en la forma de gestionar a las personas en la organización, y la mejor manera de mejorar la relación de ésta con sus empleados y de implicarse en el entorno en el que se desenvuelve. Con respecto al trabajador, dicha gestión permitiría su evolución laboral de una manera óptima, colocándolo siempre en el puesto que su nivel de competencias indicara.

Dichos manuales afirman de manera constante que la característica que identifica a la gestión por competencias es su carácter integral. Esto implica un esfuerzo considerable en cuanto a coste, tiempo y periodo de adaptación (Lawler, 1994) que no toda organización está dispuesta a hacer. Así, en ocasiones, se decide implantar el enfoque

motivaciones y las actitudes del individuo en el lugar de trabajo. 
de competencias en políticas concretas de recursos humanos como la selección y la formación (Prieto Blanco, 2007).

Pero a lo que se refieren los teóricos de la gestión por competencias es a la idílica integración de ocho grandes áreas que permitan su ideal funcionamiento, a saber: el diseño y análisis de los puestos de trabajo, la selección y la contratación, la planificación de carreras, la planificación de los relevos, la formación y el desarrollo, la remuneración, la valoración del potencial y la evaluación del rendimiento (Hooghiemstra, 1997; Carretta, 1997). Algunos autores posteriores han considerado la necesidad de agrupar dichas áreas en cinco grupos que compondrían la metodología integral de gestión por competencias (Fernández López, 2007): clasificación profesional, selección, retribución y movilidad, desarrollo profesional y evaluación y gestión del desempeño. A continuación se describirá lo que implica la gestión en cada uno de esos puntos, prestando una especial atención al desarrollo profesional, pues sería el punto en el que se incluirían tanto la valoración del potencial como la evaluación del rendimiento.

\subsection{Clasificación profesional}

La función principal de esta área es la de estructurar las funciones concretas de un trabajador o grupo de trabajadores que permitan alcanzar los objetivos definidos para sus puestos. El diseño implicará tanto a las tareas que se han de realizar como a su procedimiento y la forma en que se agruparán (Byars y Rue, 1997). El enfoque sociotécnico ${ }^{2}$ considera que en el diseño de puestos deben valorarse distintos factores con tal de evitar la rutina, lo que permite, a su vez, al trabajador familiarizarse con el puesto. Del mismo modo, hay que otorgar autonomía al trabajador para que perciba el puesto como propio, hasta el punto de que lo sienta como un vehículo que le conduzca hacia sus metas laborales deseables (Lawler, 1994; Chiavenato, 1992).

Pereda y Berrocal (2001) consideran que el diseño de puestos de trabajo implica definir los perfiles de los diferentes puestos de trabajo dentro de una organización haciendo referencia a aquellos comportamientos observables que el individuo debe desarrollar para realizar su trabajo de forma eficiente. Desde su enfoque de competencias, dichos autores consideran que el diseño de tales puestos implica la participación de los responsables de la organización y los trabajadores. Los responsables de la organización son los que deben identificar las competencias estratégicas consideradas coherentes con la misión. Respecto a los trabajadores, habrá que seleccionar un grupo representa-

2 La corriente sociotécnica en el estudio de las organizaciones establece que el sistema social que conforma al trabajador es un elemento importante a tener en cuenta a la hora de diseñar los puestos de trabajo en sistema técnico o puramente laboral. El principal precursor de dicha escuela sería el psicólogo social Eric Trist, que formó parte del Instituto Tavistock del Reino Unido. 
tivo para que escojan, entre un listado de competencias previamente identificadas por expertos en la materia, aquellas que consideren más relevantes para realizar su tarea.

\subsection{Selección de personal}

Este punto es considerado de vital importancia para una gestión integral basada en competencias, pues son las personas que entran a formar parte de las organizaciones quienes aportarán el valor distintivo. La selección de personal implica reclutamiento, selección y contratación. El perfil de exigencias del puesto guiará los procesos de selección en el camino de aportar la información más objetiva (Pereda y Berrocal, 2001).

Uno de los problemas más frecuentes al que se enfrenta un departamento de recursos humanos a la hora de seleccionar es el índice de fiabilidad y validez para predecir los comportamientos futuros de un candidato; así, existe un amplio acuerdo, en los manuales sobre gestión de competencias, en considerar las entrevistas de incidentes críticos o entrevistas conductuales estructuradas una herramienta idónea para la predicción de esos comportamientos (Boyatzis, 1982; Levy-Leboyer, 1996). Las entrevistas de incidentes críticos consisten en analizar las ideas, acciones e interacciones que han permitido al candidato realizar su tarea a lo largo de su trayectoria laboral, persiguiendo la identificación de aquellas competencias que son efectivas para conseguir un desempeño superior en un puesto de trabajo (Blanco, 2007). Por otro lado, las entrevistas conductuales estructuradas buscan recoger información de los candidatos sobre cuestiones relativas a conductas manifestadas en el pasado en circunstancias similares, basándose en la premisa de que la mejor predicción de una conducta futura es la conducta pasada en una situación similar, sobre todo cuanto más cerca esté dicha conducta del momento actual (Pereda y Berrocal, 2001).

A pesar de lo anterior, los autores de los principales manuales consideran que estas dos herramientas no cierran la puerta a otras consideradas tradicionales. Su uso junto con aquéllas permitirá aumentar el valor predictivo del proceso de selección, y, por lo tanto, la posibilidad de éxito con el candidato elegido.

\subsection{Retribución y movilidad}

Uno de los puntos de partida de los profesionales de los recursos humanos es el de considerar el alto valor del salario para atraer, motivar y retener a los trabajadores (Pereda y Berrocal, 2001; Blanco, 2007). En ese sentido, superar la tradicional forma de negociación de salarios a través de los convenios colectivos, considerados como limitados y anticuados, se convierte en una necesidad en la gestión por competencias. El salario base pierde peso para ganarlo el variable, el cual estará estrechamente ligado al logro de 
los objetivos personales y de los resultados globales de la organización. La aplicación del enfoque de competencias al sistema de retribución pretende defender la importancia de valorar el desempeño, las competencias y el potencial de los trabajadores de tal forma que se tengan en cuenta tanto el rendimiento anual del trabajador como su profesionalidad y su potencial a medio y largo plazo (Lebouin-Gelabert et alii, 1997).

En cuanto a la movilidad dentro de la gestión por competencias, lo que se pretende es definir los criterios objetivos de movilidad que marcarán la dinámica de las asignaciones de los empleados a los puestos de trabajo (Fernández López, 2006). Los procesos de movilidad quedarán determinados por la media de las diferencias de nivel que existan entre las competencias del individuo y las competencias requeridas para un determinado puesto de trabajo.

\subsection{Desarrollo profesional}

La política de movilidad se encuentra estrechamente vinculada a la planificación de carreras o desarrollo profesional, ya que no existe movilidad si no hay una visión estratégica de las carreras dentro de la organización. En ese sentido, el desarrollo profesional viene a ser la sucesión evolutiva de experiencias de trabajo de una persona a lo largo del tiempo que le permitirá adquirir, desarrollar y optimizar competencias (Arthur et alii, 1989). Una gestión integral de las competencias en la organización implicará una planificación de carreras transparente en relación con el trabajador, el cual debe conocer las opciones de carrera que para él existen, siendo asesorado sobre posibles trayectorias en función del logro de sus objetivos personales (Byars y Rue, 1997).

\subsection{Evaluación y gestión del desempeño}

La gestión del desempeño o evaluación del rendimiento es el proceso que permite evaluar los comportamientos relacionados con el trabajo y los resultados del trabajador con el propósito de medir su productividad y su posibilidad de mejora con vistas al futuro (Blanco Prieto, 2007).

Los discursos de la gestión por competencias consideran de vital importancia fijar previamente los objetivos que se han de alcanzar, abarcando no solamente los objetivos organizacionales sino también la manera de conseguirlos, estrechamente vinculada a la cultura de la empresa; en otras palabras, en la evaluación y posterior gestión del desempeño no sólo es importante evaluar el qué se hace sino también el cómo se hace (Hartle, 1996). Las organizaciones del conocimiento, aquellas que tienen en sus individuos el componente más importante de su ventaja competitiva, han comenzado a dar gran importancia a la manera en que aquellos realizan su trabajo; así, se ha convertido 
en costumbre la edición de libros, guías u hojas del buen trabajador en los que se hace referencia a los valores que se consideran importantes y necesarios en la organización, y que, por ende, ha de interiorizar y llevar a la práctica el trabajador. En ese sentido, la evaluación del desempeño es el resultado de la suma de las competencias técnicas relacionadas con el puesto que se ocupa y las competencias genéricas o los comportamientos relacionados con la ideología de la organización.

Profundizando, en primer lugar, en la evaluación de los objetivos de empresa/puesto, la dificultad estriba en la forma de trasladar un objetivo o misión a la acción. La traslación del objetivo de un puesto a la evaluación de una serie de competencias técnicas puestas en práctica supone, necesariamente, cuantificar objetivos o misiones que no siempre pueden ser evaluados numéricamente. Dicha dificultad es mucho más visible cuando la evaluación se realiza sobre competencias genéricas o comportamientos. Dichas competencias se relacionan con el saber hacer (Pereda y Berrocal, 2001), idea que encierra un alto componente de subjetividad a la hora de evaluar, ya que el individuo debe poner en práctica en su lugar de trabajo habilidades como el trabajo en equipo, la orientación al cliente o el liderazgo, y actitudes, muy difíciles de evaluar, como la simpatía, la empatía o la capacidad de escucha.

Los expertos en gestión por competencias hablan de encontrar «concreciones» de la posesión de una determinada competencia (Fernandez López, 2007). Se busca identificar escenarios críticos que permitan evidenciar que se ha logrado determinada competencia. Lo que queda menos especificado en dichos manuales es cuándo y de qué manera se realizan esas identificaciones, ya que, por lo general, en la mayoría de las organizaciones se lleva a cabo una evaluación del desempeño al año. ¿Cómo y en qué cantidad se debe realizar la observación de los escenarios críticos por parte de los evaluadores para evidenciar que se posee un comportamiento o competencia genéricos? Parece razonable pensar que existe una propensión, por parte de las organizaciones, hacia la coyunturalidad a la hora de evaluar a los trabajadores, es decir, hacia una valoración del desempeño del trabajador circunscrita al periodo de evaluación estipulado por aquéllas.

\section{Metodología y análisis}

En la medida en que el interés de la presente investigación se centra en la evaluación de empleados en organizaciones donde está implementada la gestión por competencias, se han utilizado ocho entrevistas en profundidad realizadas a directivos de recursos humanos o altos cargos de cuatro organizaciones diferentes. Estas entrevistas recogen desde el discurso más general y «oficioso» de la compañía a la que el entrevistado pertenece, hasta opiniones de tipo más personal. A continuación se detallan las entrevistas 
realizadas, según serán utilizadas en el apartado de análisis, con una breve descripción de los entrevistados:

- Entrevista n $^{\circ}$ 1: director de recursos humanos de una organización del sector del automóvil. Entrevista abierta.

- Entrevista . $^{\circ}$ 2: director de recursos humanos de una organización del sector del petróleo. Entrevista abierta.

- Entrevista . $^{\circ}$ 3: director de recursos humanos de una organización del sector de las telecomunicaciones. Entrevista abierta.

- Entrevista . $^{\circ}$ 4: encargados y encargadas de recursos humanos de una organización del sector de la selección de personal. Entrevista abierta.

- Entrevista n. ${ }^{0}$ : jefa de departamento de una organización del sector del petróleo. Entrevista estructurada, preguntas abiertas.

- Entrevista $n^{\circ}$ 6: jefe de departamento de una organización del sector del petróleo. Entrevista estructurada, preguntas abiertas.

- Entrevista n. 7 ; jefe de departamento de una organización del sector del petróleo. Entrevista estructurada, preguntas abiertas.

- Entrevista n. ${ }^{\circ}$; jefa de departamento de una organización del sector del petróleo. Entrevista estructurada, preguntas abiertas.

$\mathrm{El}$ análisis de los discursos de directivos y jefes de empresas españolas gestionadas por competencias abarca un contenido mucho más amplio que el que se pretende tratar en este artículo. El porqué de la utilización del concepto de competencia, los procesos de selección a través de ésta, la retribución o la movilidad son aspectos centrales en un análisis íntegro y amplio del uso de la noción de competencia en las organizaciones. Lo que se pretende analizar de forma concreta son los discursos que tienen relación con los procesos de evaluación a empleados gestionados por competencias. En concreto, se busca, por un lado, analizar la forma en que se realiza la evaluación y, por otro lado, el contenido y las implicaciones de ésta.

Como se comentó páginas atrás, la evaluación o gestión del desempeño abarca la valoración del trabajador tanto en sus competencias técnicas, sus conocimientos, como en sus competencias genéricas, sus comportamientos o conductas. Sala (2006) afirma que es difícil encontrar una definición clara del término competencia, por lo que considera pertinente hablar de noción más que de concepto. En ese sentido, en el discurso de los entrevistados resulta complicado encontrar una afirmación clara de lo que significaría competencia: 
De hecho, teníamos una definición de la competencia... que, espera, tengo que recordar, pero que básicamente era conjunto de conocimientos, experiencia y saber hacer ejercido en el aspecto profesional.

(Entrevista núm. 1)

De Diego et alii (2000) afirman que hablar de la noción de competencia es entrar en un «mundo diversificado y polimorfo» en el que todo tendría un posible hueco. Dicho mundo parece continuar casi diez años después en la variedad discursiva que reflejan los entrevistados a la hora de hacer referencia a la noción, la gestión o el sistema de competencias:

...el sistema de competencias viene un poco a hablar que lo que tú tienes que hacer es que la gente tenga unas competencias determinadas para poder desempeñar un puesto y que recaen más en la persona que en el puesto como tal, es decir, hay que prestarle más atención a la persona que es la que tiene los conocimientos y la experiencia y las habilidades para realizar determinadas tareas que exclusivamente el centrarte en un puesto determinado que parece que, no sé cómo decirte, que el puesto tuviera fuera una persona, y realmente los ocupantes del puesto son las personas y son los que tienen que tener las habilidades y las competencias. Entonces hay un énfasis en la persona importante y además las competencias, también en la práctica, se pueden trabajar, se pueden formar, incluso las famosas competencias genéricas o corporativas.

(Director de RR. HH., organización del sector telecomunicaciones)

En el mundo industrial, en el mundo de la empresa, pues somos más pragmáticos y hay cosas que llamamos competencias que no lo son realmente, pero bueno.

(Director de RR. HH., organización sector automovilístico)

...tenemos una definición de lo que es cada competencia, cada grado de la competencia en base a conductas que nosotros podemos observar. Entonces, cuando nosotros vemos a una persona, vemos que posee una competencia intentamos graduarla de alguna manera...

(Encargadas de RR. HH., organización de formación y selección)

Así pues, encontramos que, a pesar del paso del tiempo en la noción de competencia, ${ }^{3}$ es difícil encontrar un claro anclaje conceptual al cual referirse. Los entrevistados, en ese sentido, parecen servirse de esa difuminación en la definición para hablar de un elemento que poco tiene de novedoso con respecto a conceptos anteriores:

3 La primera referencia a la noción de competencia aplicada al rendimiento laboral la encontramos en 1974 en el artículo del psicólogo David McClleland «Testing for competence rather than intelligence», American Psychologist, $\mathrm{n} .{ }^{\circ} 28$ (1), p. 13-20. 
...quizás el asunto es nuevo con las competencias técnicas, o sea, los comportamientos, y resumo, siempre se ban tenido en cuenta, cuando una persona, parte de nuestro salario es por objetivos y en el salario por objetivos siempre, de una manera o de otra, se ha tenido regulada los objetivos técnicos a la vez que una forma de hacer las cosas, y eso ha compuesto siempre pues dinero, luego por lo tanto para mí es gestión.

(Jefa de departamento de ventas, organización sector petrolífero)

...la etapa más clara que yo puedo percibir, que sí que se hacía algún... un poco el tema de competencias pero no de una manera tan expresa, es la de una gestión pura por objetivos. Entonces, no había ese encaje de lo que se tiene o eso es lo que se quiere conseguir. O no había esa tipificación que yo conociera de qué se requiere desde el punto de vista técnico porque luego hay otras competencias que se llamaban en su día genéricas o comportamientos organizacionales y tal y también se evalúa, y también la empresa expresa qué quiere en ese aspecto...

(Jefa de departamento de tecnología, organización sector petrolífero)

Centrándonos en los tipos de competencias, encontramos, igualmente, una dificultad a la hora de unificar cómo denominar cada tipo. En apartados anteriores se ha tratado de justificar nuestra diferenciación entre competencias técnicas y competencias genéricas. A continuación, mostraremos cómo las segundas, las genéricas, son las que parecen más difíciles de precisar o denominar de una misma manera. Aquí, la pertenencia a una organización u otra implica asumir un concepto u otro:

E1: Conductuales y técnicas, también hacemos esa distinción.

I: Podríais definirlo un poco más...

E3: Conductuales, hacen referencia a las habilidades sociales, de comunicación, la conducta, el comportamiento de las personas; las técnicas van más referidas a comportamientos informáticos... más todo lo que se puede aprender, las conductuales es lo que lleva innato la persona, aunque luego pueda desarrollarlo, pero las técnicas es todo lo que se pueda ir aprendiendo, pues manejar office, manejar determinadas herramientas in formáticas, tener determinado tipo de técnicas que se pueden ir aprendiendo.

(Encargadas de RR. HH., organización de formación y selección)

Del mismo modo, una de las ideas clave de la noción de competencia es que se puede distinguir entre poseer una competencia de manera estándar o de manera superior. En realidad, los principales manuales manageriales hacen referencia a que las competencias se han de llevar a la práctica de una manera experta (Agut y Grau, 2001), pero, igualmente, surgen dificultades para encontrar un término común que defina esa competencia superior, siendo descrita de diferente manera según la organización a la que se pertenezca: 
Y el consejero metier, pues va a ser alguien, el metier es el oficio, es otra palabra francesa... que es va a estar pegado al terreno, va a ayudar al piloto de competencias que es un directivo y que tiene muchas cosas que ver y que a lo mejor no puede llegar a fondo en ciertos aspectos y que le va a permitir animar pues algunos foros, porque esto tiene que basarse en una animación donde puede, puede ir viendo todo lo que tiene el grupo, ¿vale?

(Director de RR. HH., organización sector automóvil)

...en áreas como tecnología tenemos científicos investigadores que son auténticos gurús dentro del sector petrolero a nivel mundial, son personas que escriben libros, dan conferencias, muchos de ellos trabajan en las universidades como profesores, etcétera. Y ésos son los gurús.

(Director de RR. HH., organización sector petrolífero)

...pues lo que te decía, o sea que, yo qué sé, hubo personas que en ocho o diez competencias tenían un cinco, por poner el caso más extremo, que es un referente mundial en esta organización y fuera de ella en esa competencia, entonces se pidió a los responsables que revisaran esa evaluación con unos criterios de decir... no sé si conoces la escala...

(Jefa de departamento de ventas, organización sector petrolífero)

El objetivo es el compromiso y la cible es un poquito más allá del compromiso. $\mathrm{O}$ sea, es una forma de decir, bueno lo mínimo es el objetivo, eso no puede fallar, y lo ideal es alcanzar la cible. La primera aceptación que tiene es un poco el ideal de competencias.

(Director de RR. HH, en organización sector automóvil)

Adentrándonos en la evaluación de las competencias, encontramos una idea central que recorre el discurso de la totalidad de los entrevistados. Con las competencias técnicas se evalúa la parte objetiva en la realización de la tarea, sería el saber, lo que en apartados anteriores hemos denominado el qué. Así, casi la totalidad de los entrevistados relacionan la idea de competencias técnicas con la de objetividad:

...pero bueno, ha habido un esfuerzo de intentar objetivizar siempre que se ha podido, basándose en documentos, procesos, indicadores, cosas concretas y cuantificables...

(Director de RR. HH., organización sector automóvil) 
Es una escala que, a la hora de evaluar, te permite establecer un mismo criterio, un único criterio para toda la compañía, y eso es muy importante porque si luego a las personas, se va a utilizar toda esta información de competencias para tomar decisiones a nivel de clasificación profesional, en cuanto a formaciones, desarrollos, etcétera, tiene que estar todo lo más objetivado posible...

(Director de RR. HH., organización sector petrolífero)

En esta hoja, como puedes ver, al lado hay un criterio de validación de la competencia. A ver, esto es un poco una explicación más detallada, ¿"controla el seguimiento de fabricación»? significa que "el operario aplica la totalidad de los procedimientos y las reglas definidas en el plan de aseguramiento de calidad del perímetro que le concierne», para explicar un poco más. El criterio de evaluación es para ayudar a la persona que le tiene que evaluar a... a ... a tener que, sobre qué evidencia se basa decir si la tiene o no la tienes, es decir, que no sea algo subjetivo.

(Director de RR. HH., organización sector automóvil)

...pero siempre hay que tratar de desarrollar herramientas que tengan componentes lo más objetivos posible para ser lo más justos posible.

(Director de RR. HH., organización sector petrolífero)

Es curioso cómo, a pesar de esa tendencia a vincular competencia genérica con objetividad, al final se demanda al individuo, como aparece en el extracto siguiente, una especie de reflexión interior objetiva, afirmación que parece casi una tautología cuando es el individuo el que ha de ver, de forma objetiva y en su interior, si posee en el grado que se le pide cierta competencia:

..y además es una forma de objetivizarlo realmente, cuando tú estás solicitando a una persona que realice determinadas funciones o que realice determinadas cosas y las hace o no las haces es una forma de decir «bueno, realmente tu función y lo que se espera de ti es esto con esta competencia con este grado», tú sabrás si lo tienes o no, es algo objetivo entonces realmente una persona puede decir "pues sí, tienes razón»o «no, no tienes razón», «llego o no llego»...

(Encargadas de RR. HH., organización de formación y selección)

En cuanto a las competencias generales o los comportamientos, ocupan un porcentaje menor con respecto a las competencias técnicas en el resultado final de la evaluación: 
Debes tener en cuenta que no tienen el mismo peso las competencias técnicas y los comportamientos. Podríamos decir que en el resultado de la evaluación las competencias como tal suponen entre un $70 \%$ y un $80 \%$, mientras que los comportamientos vendrían a ser un $20 \%$ o un $30 \%$...

(Jefe de departamento, organización sector petrolífero)

En cambio, otros jefes que hablan de su experiencia en la evaluación de sus trabajadores revelan el carácter variable que pueden tener los comportamientos en el resultado de la evaluación del rendimiento. La importancia de las competencias genéricas parece depender más del tipo de jefe y del tipo de relación que éste mantenga con su empleado directo, incluso de la carga de trabajo que el proceso de evaluación conlleve:

Las genéricas se han transformado en comportamientos, esos comportamientos se evalúan anualmente, bueno, hay un seguimiento en junio y una evaluación anual, y tiene un peso inespecifico sobre la evaluación final de desempeño inespecificado, o sea, que no es medido "esto es el 20\%», no, cada uno lo aplica como mejor sabe, cada gestor lo aplicamos como podemos.

(Jefa de departamento de ventas, organización sector petrolífero)

Con los comportamientos, las compañías inciden en el cómo se realizan las tareas, el saber hacer. Como vimos anteriormente, este aspecto se ha convertido en central a la hora de considerar al individuo como el elemento clave a la hora de conseguir la ventaja competitiva en el mercado. A pesar de que no quede muy claro cuál es su peso a la hora de la evaluación final, las competencias de tipo conductual son las que, cada vez más, se tienen en cuenta a la hora de evaluar:

...y luego en el trabajo diario, en la gestión por compromisos se ponen en juego y se le piden a cada persona evidencias y lo que intentamos también los gestores es que se premien también esos comportamientos....

(Jefa de tecnología, organización sector petrolífero)

... se le dice «mira, has pasado la formación, pero al trabajar esto creemos que tienes un potencial pero, por ejemplo, tienes que seguir comunicándote más, por ejemplo, asertivamente con el resto de tus compañeros, ahí tienes que seguir»...

(Encargadas de RR. HH., organización de formación y selección)

...Y entonces un poco se descubre que algunas de las características tienen que ver pues cómo la cultura japonesa aborda indudablemente el trabajo en equipo, perdón, el trabajo productivo y tiene que ver también pues cómo conciben el trabajo en equipo, la 
colaboración y una serie de cosas y entonces viene el dar importancia a ese tipo de aspectos que hasta ahora se les daba poca importancia, es decir, lo único que se pensaba es que la gente tenía que tener competencias técnicas pero no necesitaban otro tipo de competencias, $y$ muchos problemas tenian que ver pues con que la gente no se comunicaba, ni transmitía la información, ni babía feedback y había muchos otros problemas que no eran puramente derivados de la técnica, sino que era un poco más de las relaciones humanas.

(Director de RR. HH., organización sector telecomunicaciones)

...yo le llamo el cómo hacer las cosas y eso está recogido y es fundamental tanto en la selección de las personas que entran a trabajar en esta organización, porque en los comportamientos hay comportamientos clave, bay un set de comportamientos con su definición pero luego hay los denominados comportamientos clave...

(Jefa de departamento de tecnología, organización sector petrolífero)

...a gestión por compromisos, el desempeño de las personas, o sea, evalúas a las personas en su desempeño en el año, que son las funciones de responsabilidades y proyectos y los comportamientos, que son las competencias genéricas, eso mide su desempeño en el año y esto es una puntuación.

(Jefa de departamento de ventas, organización sector petrolífero)

Los comportamientos incluso pueden llegar a estar mucho menos explicitados en relación con el trabajador, es decir, que no exista una transparencia de la organización con aquél para valorar las competencias genéricas, pero que su puesta en práctica sea condición necesaria no tanto con vistas a su evaluación concreta como en relación con el progreso de su proyecto laboral en la organización:

Entonces, eso es un tema que se empieza a dar cuenta, las empresas de que le tienen que pedir al ingeniero no solamente que sepa mucho de la máquina, sino que sea capaz de gestionar un proceso, capaz de coordinarse y cooperar con otra persona para conseguir fines comunes..., y para eso se requieren otras muchas habilidades que no son exclusivamente las técnicas.

(Director de RR. HH., organización sector automóvil)

La tensión resultante del qué y el cómo, debido a que se sitúan en parámetros opuestos (el primero cuantificable $y$, por lo tanto, objetivable, y el segundo referido a cualidades y, por lo tanto, subjetivo) pero que, a su vez, no se reconocen como incompatibles, daría como resultado una falta de criterios claros a la hora de evaluar al empleado. Así, el protagonista final de la evaluación se atiene a variables que están fuera de su control, comités de validación, coyuntura económica, etc.: 
Eso se analiza, se analiza con un sistema, tenemos muchos informes que sacamos información de aquí, y uno de ellos es identificar por un lado esas discrepancias entre diferentes evaluadores dentro de una misma competencia, cuando una persona; o a veces falta de contraste... Todo eso se analiza dentro de RR. HH., se generan unos informes que se presentan a esos comités de validación del negocio donde están los directores, donde está... y donde se analiza esa información. Se analizan juntos a otros informes que es una tendencia de evaluadores, pues se analiza cuando una persona, pues el número de personas que ha evaluado un señor determinado, la media que ha utilizado, la moda, etcétera...

(Director de RR. HH., organización sector petrolífero)

El problema que conlleva eso, pues, como lo hemos visto, es que puede quedar algo más subjetivo y eso hace que podamos tener un problema a la hora de validar el nivel de competencias, me explico, yo soy jefe de taller en pintura, tengo mi visión, yo veo estoy lo entiendo a mi manera y según mi referencial, mis creencias, mis valores, lo que tengo en mi entorno, mi historia; entonces yo voy a ver a este jefe de unidad [JU] y voy a decir «buah, ¿éste controla los costes? No controla, tiene un seguimiento pero se sigue gastando lo que se sigue gastando, le pongo un $\mathrm{B}$ » $\mathrm{y}$ me voy a lo mejor uno de montaje y dice «tiene el indicador, con que tenga el indicador mucho es, le pongo un D», y están los dos JU haciendo lo mismo pero resulta que su línea jerárquica no les está evaluando igual...

(Director de RR. HH., organización sector automóvil)

Un sistema que permite interrelacionar una cosa con otra a través de una evaluación de competencias, es decir, la persona se evalúa, la evalúa su jefe, se evalúa la persona con una autoevaluación y nos permite hacer ese mache. Todo esto está lógicamente relacionado con las políticas, sistemas y planes de recursos humanos que están instaurados en la compañía a nivel corporativo y, siempre, establecido por la misión, visión y objetivos estratégicos que establece la organización a nivel mundial.

(Director de RR. HH., organización sector petrolífero)

Las competencias técnicas y las generales se encuentran enfrentadas en cierta medida por el deseo por parte de los directivos y jefes de introducirlas en un mismo saco en la evaluación del desempeño del trabajador. Las referencias constantes al carácter subjetivo de las competencias generales o comportamientos también acentúan dicho enfrentamiento. La tensión entre ambos tipos de competencias parece resolverse con un acotamiento de la subjetividad de una manera un tanto simple, pues las cualidades o los aspectos más actitudinales de un individuo nunca podrán ser reducidos a la cuantificación necesaria que implica la evaluación. Las competencias generales o com- 
portamientos devienen un espacio compartido por evaluador (jefe) y evaluado que inexorablemente implica divergencia; el evaluador se estaría comprometiendo a un acto intersubjetivo (Husserl, 1982), pues es consciente de las implicaciones diferentes de dichas competencias: pretender objetivar y cuantificar aquello subjetivo e incuantificable.

\section{Conclusiones}

A lo largo de las anteriores páginas se han explicitado, a través del discurso, los mecanismos y procesos que se dan en la evaluación del desempeño en diferentes organizaciones gestionadas por competencias. La información ha provenido de lo que podríamos denominar «el poder organizacional», directivos de recursos humanos y jefes de departamento. Si bien su discurso comparte características comunes, existirían muchas especificidades en función del cargo ocupado y de la empresa a la que se pertenezca.

Los directivos de recursos humanos, con una visión mucho más estratégica y amplia, definen el concepto de competencia de una manera más estratégica. Su discurso se enmarca, en numerosas ocasiones, en el deber ser, mientras que los jefes y encargados, además de tener un discurso más heterogéneo en función de la realidad con la que conviven día a día, explicitan más el ser, lo que les sucede en el quehacer diario. Consecuentemente, este discurso plantea con mayor claridad la tensión que provoca valorar competencias que no responden a la puesta en práctica de un saber.

Realizar un análisis completo de lo que implica la evaluación por competencias de los trabajadores nos llevaría necesariamente a analizar el discurso de estos últimos. Su discurso permitirá dialogar con el de sus jefes y directivos y enfatizar lo que en este artículo sólo se atisba: la no neutralidad de la evaluación de sus competencias. Esta falta de neutralidad en la evaluación sería otra muestra de la exigencia de una disponibilidad total para el trabajo con una escasa garantía de estabilidad, lo que mercantiliza los reductos más personales del individuo, sus recursos afectivos y sociales (Crespo, 2009).

Un análisis aún más global y necesario se derivaría de analizar no sólo el discurso en el área de evaluación de desempeño, sino en todas las áreas a las que cualquier manual managerial sobre gestión por competencias hace referencia. Este estudio global, que tendría que tener en cuenta tanto el discurso de los empleadores como el de los empleados, permitiría arrojar luz a una gestión que, a pesar de ser una de las gestiones referentes en las organizaciones del siglo XXI, sigue sin ser analizada desde «dentro» de la organización. Este análisis permitiría contrastar si los numerosos enunciados presentados en multitud de manuales manageriales como positivos lo son verdaderamente para todos los miembros de la organización o si, por el contrario y como se ha intentado mostrar en este texto, están escritos para un grupo muy concreto y pequeño dentro 
de la organización al que se otorga mayor capacidad de agencia, e, inevitablemente, conllevan la fragilización y debilitación de la mayoría, es decir, de los trabajadores.

\section{Bibliografía}

Agut, S. y Grau, R. (2001). Una aproximación psicosocial al estudio de las competencias. Castellón: UJI.

Arthur, M. B.; May, D. T. y Lawrence, B. S. (1989). Hanbook of career Theory. Cambridge: Cambridge University Press.

Blanco Prieto, A. (2007). Trabajadores competentes. Introducción y reflexiones sobre la gestión de recursos humanos por competencias. Madrid: ESIC.

Boyatzis, R. (1982). The competent manager: a model for effective performance. Nueva York: Wiley \& Sons.

Brunet, I (2005). «Gestión por Competencias», en VV.AA. Competencias, Igualdad de oportunidades y eficacia de la Formación Continua. Madrid: Fundación Tripartita.

Byars, Ll. y Rue, L. W. (1997). Gestión de recursos bumanos. Madrid: McGraw-Hill.

Carreta, A. (1997). «Aplicaciones en la planificación de recursos humanos», en M.M. Dalziel; J.C. Cubeiro, y G. Férnández (comps.). Las competencias: clave para una gestión integrada de los recursos humanos. Bilbao: Deusto Ediciones.

Chiavenato, I. (1992). Administración de recursos humanos. México: McGraw-Hill.

Crespo, E. (2009). »Las morales del trabajo», en E. Crespo; C. Prieto y A. SerraNo (eds.). Trabajo, subjetividad y ciudadanía. Paradojas del empleo en una sociedad en transformación.

Crespo, E.; Revilla, J. C. y Serrano, A. (2005). «La psychologisation politique du travail», en A. Dorna (ed.). Psychologie Politique. París: L'Harmattan.

Dalziel, M. M.; Cubeiro, J. C. y Fernández, G. (1996). Las competencias: clave para una gestión integrada de los recursos humanos. Bilbao: Deusto.

Fernández López, J. (2006). Gestión por competencias. Un modelo estratégico para la dirección de Recursos Humanos. Madrid: Prentice Hall, Pearson.

Hooghiemstra, T. (1997). «Gestión integrada de recursos humanos», en M.M. Dalziel; J.C. Cubeiro, y G. Férnández (comps.). Las competencias: clave para una gestión integrada de los recursos humanos. Bilbao: Deusto Ediciones.

Husserl, E. (1982). La idea de la fenomenología: cinco lecciones. Madrid: Fondo de Cultura Económica.

Lawler, E. E. (1994). «From Job-Based to Competence-Based Organizations». Journal of Organizational Behavior, 15, (1):3-15.

Lebouin-Gelabert, Ch.; Hoffmann-Hervé, M. y Aubert, N. (1997). Diriger et motiver. París: Les Éditions d'Organisation. 
Levy-Leboyer, C. (1996). Gestión de las Competencias. París: Gestión 2000.

McClelland, D. (1973). «Testing for competence rather that intelligence». American Psychologist, 28 (1): 13-20.

Pereda, S. y Berrocal, F. (2001). Gestion de recursos bumanos por competencias. Madrid: Centro de Estudios Ramón Areces.

SAlA, G. (2006). «Cambio de noción. La gestión por competencias en el sector bancario español». Trayectorias, 22: 21-33.

Serrano, A. y Crespo, E. (2002). «El discurso de la Unión Europea sobre la sociedad del conocimiento». Revista Española de Investigaciones Sociológicas, 97: 189-210.

Spencer, L. y Spencer, S. M. (1993). Competence at work, models for superior formante. Nueva York: Wiley $\&$ Sons.

Tovar, F. J. y Revilla, J. C. (2009). «An alternative view of competence-based Management», en P. Koistinen; L. Mósesdóttir y A. Serrano Pascual (eds.). Emerging Systems of work and welfare. Bruselas: Peter Lang. 